\title{
Preference-based User Learning using Social Media Marketing for Distributed Cloud Information Retrieval
}

\author{
Ezhumalai.P $\mathbf{P}^{\mathbf{1}}$, Vinoth. $\mathbf{V}^{\mathbf{2}}$ \\ ${ }^{1}$ Assistant Professor, Department of Computer Science \& Engineering, Christ Institute of Technology, \\ Pondicherry University, Puducherry, India \\ ${ }^{2}$ Scholar, M.Tech, Computer Science \& Engineering, Christ Institute of Technology, \\ Pondicherry University, Puducherry, India \\ 1ezhusuccess16@gmail.com, ${ }^{2}$ vvinoth777be@gmail.com
}

\begin{abstract}
Information retrieval plays a vital role in social media marketing to promote the product or service in the cloud computing environment. The main issue in social media marketing is to analyze and provide information according to users' needs. Generally, the user preference is analyzed only by the initial query which is not sufficient for the recommendation. In this paper, Ontology-based Information Retrieval (OIR) framework is designed for retrieving metadata from users to promote social media marketing (SMM) system. OIR collects the user searching information and builds the training process to improve social media marketing. This reduces the computational cost and time by automatic learning from the user's search.
\end{abstract}

Keywords: Support Vector Machine algorithm, Information Retrieval, Social Media marketing, Ontology model.

\section{INTRODUCTION}

The powerful computing capacity, flexible service model and low cost of cloud computation have attracted many individuals, companies, government, and other users. At the same time, it has a great influence on the IT industry and can thoroughly change the architecture and operation mode. Although cloud computation provides convenience, it is still confronting numerous security threats and challenges. Information retrieval is one of the main services in cloud computation. It can denote the database as $\mathrm{n}$-bit data base $\mathrm{x}=\mathrm{x}_{1}, \mathrm{x}_{2}, \ldots \mathrm{x}_{\mathrm{n}}$ and they are stored in the server. It allows fetching $\mathrm{i}^{\text {th }}$ bit while we do not intend anyone to know which bit. It can define a scheme possessing this property as Information Retrieval [1]. Several applications have been proposed for Information Retrieval, including online census information, real-time stock search and so on. It is especially suitable for anonymous post retrieval [2].

There are two classes in information retrieval they are, computational private information protocol (C-PIR) if an adversary can deal with an encryption scheme, so it can get the user query detail. That is to say, its security bases on the encryption scheme. Second is informationtheoretic-IR the server is unable to determine any information about your query even with unbounded computing power. It is hard to achieve in a single-server case, while for the multi-server case it is practicable, e.g.,
Multi-servers Information Retrieval [3]. The traditional solution for Information Retrieval is to download the whole database and search for the desired bit or file. The goal of Information Retrieval is to guarantee database security and reduce communication costs and time. In the cloud environment, it is impractical to solve a problem like this, and this will greatly consume the network bandwidth with poor data delivery.

Social Media Marketing makes use of social media sites to raise visibility on the Internet and to promote products and services. Social media sites are useful for building social (and business) networks, and for exchanging ideas and knowledge [4]. Social media networking is part of a trend known as Web 2.0, which refers to changes in the way users and software developers use the Web. It is a more collaborative use of the Web that enhances creativity and knowledge exchange. It is a more interactive and user-driven way to help users participate and collaborate over the Web through open applications and services. It is critical, therefore, that content is accessible to the user, the user should be able to create, share, remix, and repurpose content. Technologies that are accessible and affordable like Ajax ( a method of building interactive applications for the Web that processes user requests immediately) and RSS (Really Simple Syndication) support Web 2.0 principles such as "user empowerment". 


\section{International Journal of Computing and Mathematics}

Vol: 2 Issue: 1 March 2018

Social Media Marketing uses podcasts, wikis, blogs, folksonomies, online videos, photo sharing, news sharing, message boards, and posts on social networking sites to reach a large or targeted audience.

Traditionally, the retrieval is carried out from the cloud storage server as the raw data without any learning process. In proposed framework is the combination of several methodologies which are mentioned as follows:

1. Collection of user preference data about the product.

2. Information retrieved for the requested data.

3. Constructing Ontology learning process.

4. Recommendation product.

\section{RELATED WORKS}

Fan Wang et al., [6] Adaptive User Preference Elicitation scheme based on the Collaborative filtering (CF) algorithm for location recommendation is proposed. User preference is categorized as user static preferences and user dynamic preferences. The former is estimated based on location category information and historical ratings. Meanwhile, the latter is evaluated based on geographical information and a two-dimensional cloud model. To predict user preferences of new locations more precisely, the scheme integrates the similarity of user static preferences, user dynamic preferences and social ties into CF algorithm.

Jiadi Yu et al., [7] Top-K multi-keyword retrieval with two-rounded searchable encryption (TRSE) is used to avoid data leakage. TRSE is the combination of both the vector space model and the homomorphic encryption process is used. The vector space model helps to provide sufficient search accuracy and homomorphic encryption that enables users to involve in the ranking, while the majority of computing work is done on the server-side by operations only on the ciphertext. The retrieval process is the communication takes place in a cloud server and data user.

Sangwon Lee et al., [8] the relationships among usability/aesthetics features, perceived usability/aesthetics, and user preference through an experiment using four simulated systems with different levels of usability and aesthetics. The results showed that, before actual use, user preference was significantly affected by the differences in aesthetics but marginally affected by the differences in usability. On the other hand, after actual use, user preference was significantly influenced by the differences in both usability and aesthetics. Regardless of the occurrence of actual use, user preference was highly correlated with both perceived usability and perceived aesthetics, which were strongly interrelated. Finally, actual use had a significant effect on perceived usability, perceived aesthetics, and user preference.

Yue-Shan Chang et al., [9] Ontology-based agent generation framework for information retrieval is proposed for the cloud environment. The information is retrieved by submitting the user request as a flat text and it automatically deduces the Reasoning Agent (RA) based on predefined ontology and reasoning rule. Later it is translated into Mobile Information Retrieving Agent Description File (MIRADF) that is formatted in a proposed Mobile Agent Description Language (MADF). A generating agent, named MIRA-GA, is also implemented to generate a MIRA according to the MIRADF. Additionally, a Knowledge synthesis agent is designed to collect and synthesize the information from the clouds.

Qin Liu et al., [10] The keyword-based file retrieval scheme is used with the Aggregation and Distribution Layer (ADL). This efficient information retrieval for the ranked query (EIRQ) is used to reduce querying costs in the cloud. Queries are classified into multiple ranks, where a higher-ranked query can retrieve a higher percentage of matched files. ADL layer lies as the intermediate between the users and the cloud. It aggregates query from multiple users and sends as a combined query to the cloud. The degree of aggregation can be controlled by the time-out framework to meet the given processing delay requirement. The probability of a file being returned by the highest rank of queries matching this file is less.

\section{PROPOSED SYSTEM}

The idea behind the proposed approach is to use the different methods to provide an efficient information retrieval system in the cloud computing environment. The aim is to start with tracing the user behavior to build the ontology learning process.

The proposed methodology is divided into two phases.

1. Collection of user preference data about the product.

2. Information retrieved for the requested data.

3. Constructing Ontology learning process.

4. Recommendation product. 


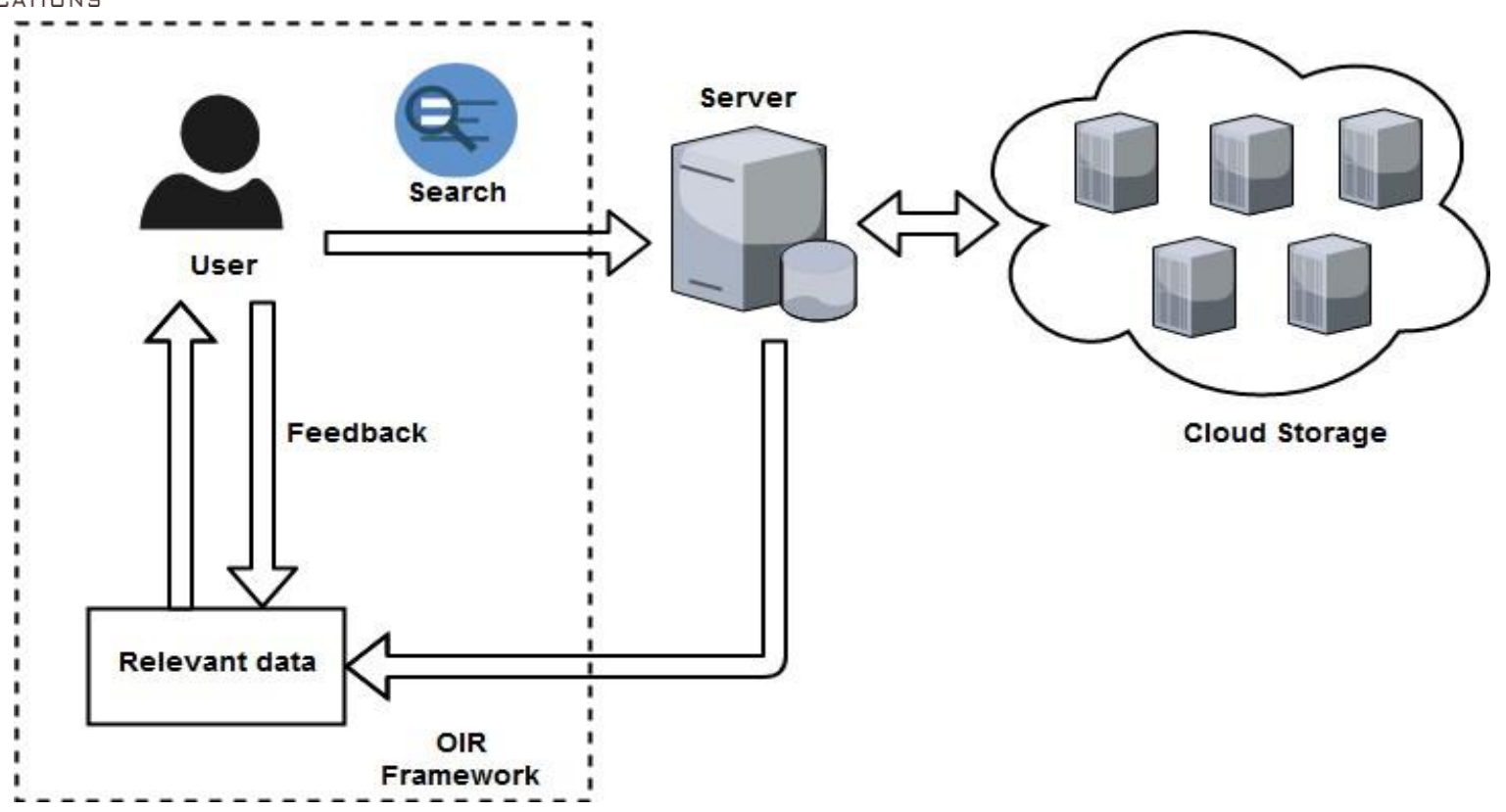

Figure 1. Represents the Ontology Information Retrieval Framework

\section{Users Preference}

It includes a collection of feedback from the users and used it as a guide when deciding the class for the future user search. The user preference includes the collection of data from user likes, user searching keywords, user's feedback, and so on. These data are used to construct the ontology based on the

\section{- Concept learning}

More details about the suggested concepts are the new version has extended keyword list describing suggested concepts. Generate suggestions only when explicitly asked and now the user must click a button to generate a suggestion list. The user knows what sub-concept to add but the system does not suggest it, now the user can generate concept suggestions by providing a query (for this task we used active learning)

\section{- Concept management}

How can the user move a sub-concept? This is already possible in the previous version by adding and removing relations; this is greatly simplified in the new version. The system suggests a sub-concept which is not related to the selected concept and added option to prune the suggested sub-concept which also removes related documents from the selected concept

\section{Information Retrieval}

The information retrieved for the user search is collected for the learning process. In Information Retrieval protocol, a server possesses a database and a user would like to retrieve the $i$-th element of it. At the end of the protocol, the user will get the element and the server will know nothing about $i$.

The protocol can be summarized as follows. Let $i$ be the index requested by the user for an $n$-tuple $\left(\mathrm{x}_{1}, \mathrm{x}_{2}, \ldots \ldots \mathrm{x}_{\mathrm{n}}\right)$. First, the user generates a query $\mathrm{Q}=\left(\mathrm{q}_{1}, \mathrm{q}_{2}, \ldots \ldots . \mathrm{q}_{\mathrm{n}}\right)$, Where,

$q j=\{\operatorname{Enc}(0)$ for $j \neq i$ Enc(1)for $j=i$

and sends it to the server. Second, the server computer and sends back the response $\mathrm{R}=\sum j=1^{n}(\mathrm{qx})_{\mathrm{j}}$. Finally, the user decrypts the response and gets the information requested $\operatorname{Dec}(\mathrm{R})=\mathrm{x}_{\mathrm{i}}$. Here, Enc and $\operatorname{Dec}$ correspond to the encryption and decryption function of an additive homomorphic scheme (e.g. Paillier's), respectively.

\section{Learning Process}

Knowledge discovery techniques based on machine learning can be applied to Ontology learning. Some of those techniques can be described as supervised learning, unsupervised learning, semi-supervised learning, and active learning. Support Vector machines implement complex decision rules by using a nonlinear function - to map training points to a high-dimensional feature space where the labeled points are separable. A separating hyperplane is found which maximizes the distance between itself and the nearest training points(this distance 
is called the margin). The hyperplane is represented as a linear combination of the training points. Theoretical results exist from VC theory, which guarantees that the solution found will have high predictive power, in the sense that it minimizes an upper bound on the test error (a survey covering the generalization power of SV machines).

\section{PERFORMANCE EVALUATION}

We have evaluated the performance of the OIR framework on several standard classification datasets, both artificial and real. 200 users searched for 1762 products and given feedback for it under the OIR framework.

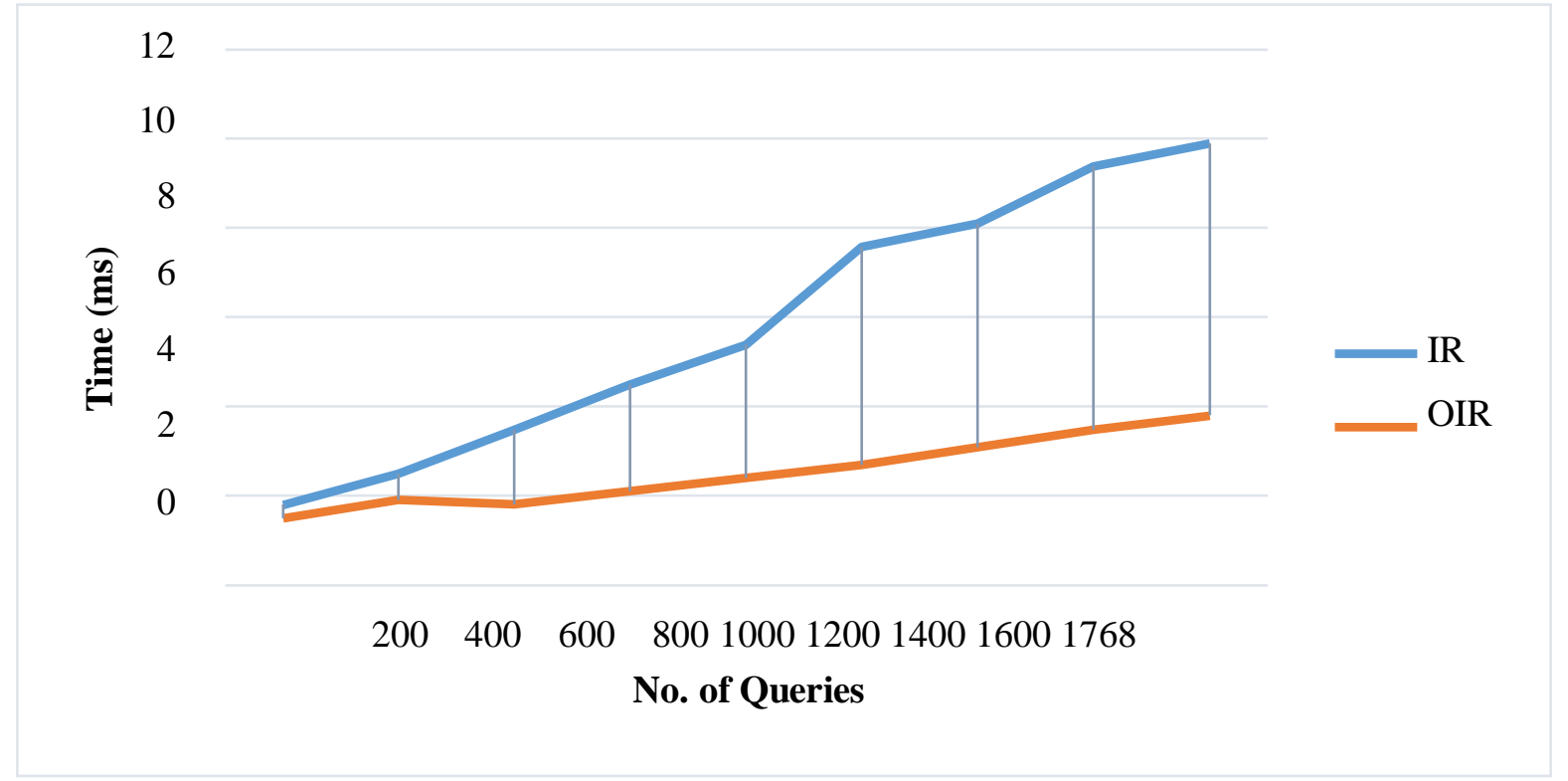

Figure 2. Represents the time taken to retrieve the data by comparing OIR technique with IR technique

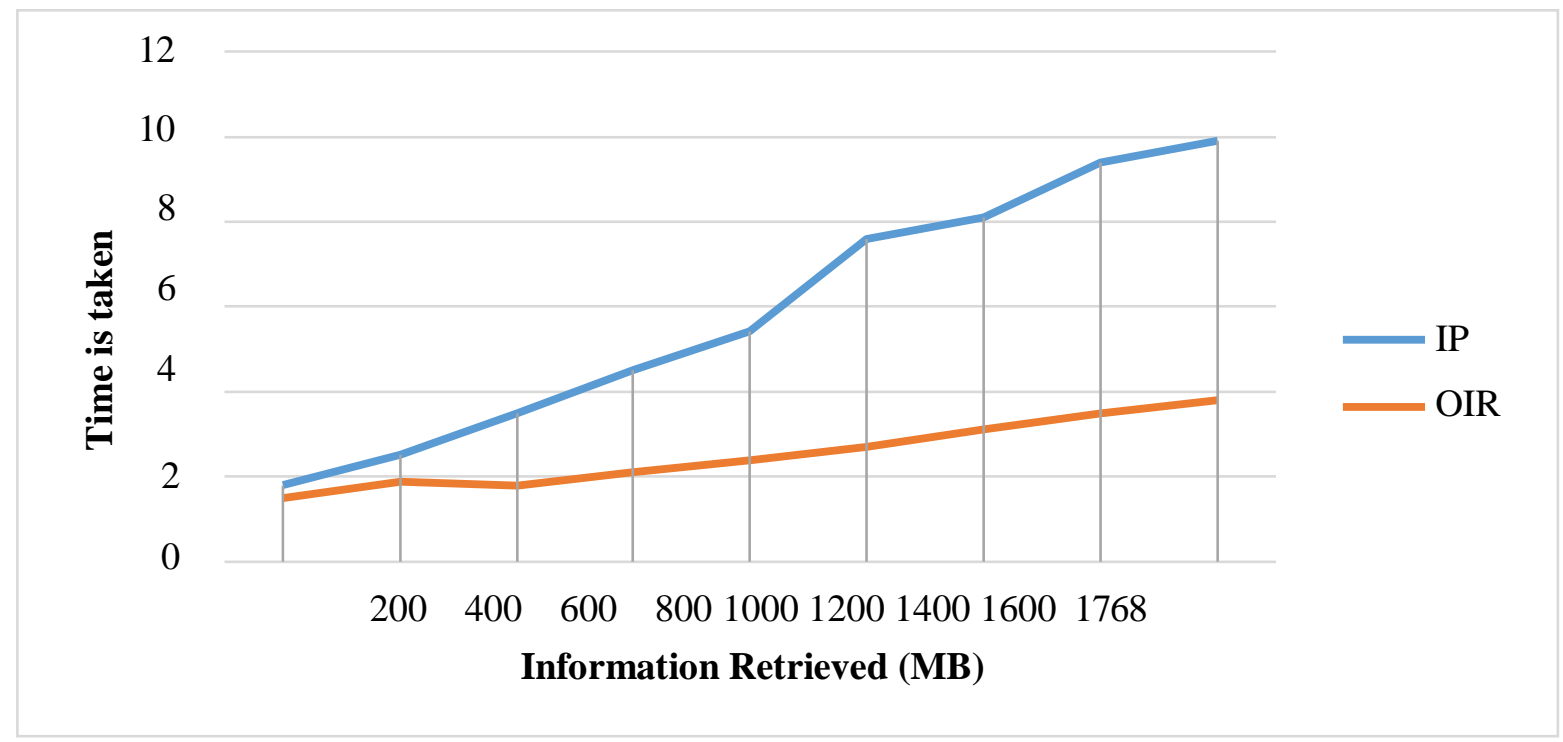

Figure 3. Represents the time taken for SVM learning from ontology data in OIR technique with Naïve Bayes User preference learning 


\section{International Journal of Computing and Mathematics}

Vol: 2 Issue: 1 March 2018

\section{CONCLUSION}

OIR framework provides a new approach to manage information and processes by learning from user preference. Ontology learning is one of the phases for managing information in the OIR framework. To build the framework, it is necessary to trace the user preference, keyword searched, data retrieved, and handling instances. Traditional methods of information retrieval services are time-consuming and expensive. From this point of view, a framework is suggested to automate the different tasks, like learning from user preference, information retrieval from cloud storage, adding metadata with the Ontology.

\section{REFERENCES}

[1] B. Chor, O. Goldreich, E. Kushilevitz, and M. Sudan,"Private information retrieval", In 36th Annual Symposium on Foundations of Computer Science, Milwaukee, Wisconsin, pp. 23-25, 1995.

[2] Zhen Yang, Jiliang Tang, and Huan Liu, "Cloud Information Retrieval:Model Description and Scheme Design", In IEEE Translations and content mining, Vol. 6, 2018.

[3] KazutoshiSumiya, Daisuke Kitayama,Naiwala P. Chandrasiri, "Inferred Information Retrieval with User Operations on Digital Maps", IEEE Internet Computing, Vol. 18, Issue 4, 2014.

[4] Chi-Hsiung Chen, Yi-Wen Chen, "The study of social media design marketing status", In IEEE International Conference on Applied System Invention (ICASI), pp. 807- 810, 2018.

[5] Magdalini Eirinaki, Malamati D. Louta, Iraklis Varlamis, "A Trust-Aware System for Personalized User Recommendations in Social Networks", In IEEE Transactions on Systems, Man, and Cybernetics Systems, Vol. 44, Issue 4, pp. 409-421, 2014.

[6] Fan Wang, Xiangwu Meng, Yujie Zhang, “An Adaptive User Preferences Elicitation Scheme for Location Recommendation", In IEEE Chinese Journal of Electronics, Vol. 25, Issue 5, 2016.

[7] Jiadi Yu, Peng Lu, Yanmin Zhu, GuangtaoXue and Minglu Li, "Towards Secure Multi-Keyword Top-k Retrieval over Encrypted Cloud Data”, In IEEE
Transactions on Dependable and Secure Computing, Vol. 10, Issue 4, 2013.

[8] Sangwon Lee, Richard J. Koubek, "Understanding user preferences based on usability and aesthetics before and after actual use", vol. 22, Issue 6, 2010.

[9] Yue-Shan Chang, Chao-Tung Yang, Yu-Cheng Luo, "An Ontology based Agent Generation for InformationRetrieval on Cloud Environment", In Journal of Universal Computer Science, Vol. 17, No. 8, 2011.

[10] Qin Liu, Chiu C. Tan, Jie Wu, and Guojun Wang, "Efficient Information Retrieval for Ranked Queries in Cost-Effective Cloud Environments", In Proceedings IEEE INFOCOM, 2012. 